\title{
Caractérisation de la diversité d'une population de vignes sauvages du Pic Saint-Loup (Hérault) et relations avec le compartiment cultivé
}

\author{
Patrice This*, Catherine Roux, Pierre PARra, René Siret, \\ Thibaut Bourse, Anne-Françoise AdAm, Michel Yvon, \\ Thierry Lacombe, Jacques David, Jean-Michel Boursiquot
}

Institut national de la recherche agronomique - ENSA.M, 2, place P. Viala, 34060 Montpellier Cedex 1, France

\begin{abstract}
Résumé - Vitis vinifera est la seule espèce endémique de vigne en Europe. Le compartiment sauvage Vitis vinifera subspecies silvestris a subi une forte érosion et seuls quelques peuplements subsistent encore actuellement. Afin de mieux connaître les relations entre les compartiments sauvage et cultivé, nous avons engagé une étude de la diversité de populations naturelles. Une population de vignes sauvages du Pic Saint-Loup (Hérault) a ainsi été caractérisée. La cartographie de cette population constituée d'une quinzaine d'individus ainsi que quelques caractéristiques biologiques sont présentées. Le caractère dïoique de la plupart des accessions a été confirmé avec une présence importante de pieds mâles (58\%). La diversité des individus est discutée et comparée à l'aide de différentes approches : étude morphométrique des feuilles (ampélométrie), polymorphisme moléculaire à l'aide de dix locus microsatellites, degré de tolérance à l'oïdium (Uncinula necator) et au phylloxera. Dans le cas des marqueurs moléculaires, les données sont comparées aux résultats obtenus sur un échantillon incluant les principaux cépages cultivés et autochtones présents dans la région. L'analyse en composantes principales réalisée à partir de ces données fait apparaître une séparation très nette des individus sauvages par rapport aux individus cultivés.
\end{abstract}

silvestris / lambrusques / ampélométrie / microsatellites / résistance

\begin{abstract}
Characterization of genetic diversity in a population of wild grapes from Pic Saint Loup area and it's relationship with the cultivated grapes. Vitis vinifera is in fact the only species of grapevine endemic in Europe. The wild compartment Vitis vinifera subspecies silvestris suffers of important erosion and just a few populations are still subsisting today. To better know the relations between
\end{abstract}

* Correspondance et tirés-à-part

E-mail: this@ensam.inra.fr 
the wild and the cultivated compartment we began a study about the diversity of the natural populations. For that, a group of about fifteen wild vines being situated on the Pic Saint-Loup (Hérault, France) has been characterized. The mapping of the population and some biological characteristics are presented. We verified that most of the accessions owned effectively flowers showing separated sex. The majority of the vines $(58 \%)$ were male. The diversity inside the population is discussed and compared through different approaches. We analyzed the morphology of the accessions by ampelography and measurement of the leaves. The molecular polymorphism of the individuals has been evaluated with ten microsatellites loci and we checked the degree of tolerance of the accessions to powdery mildiou (Uncinula necator) and to phylloxera. With the molecular markers we also compared the data of the wild population to the results obtained on a sample of old varieties originated from the area and of the actual main cultivated varieties. The principal component analysis based on these molecular data shows finally a very clear division between the wild accessions and the cultivated varieties.

Vitis v. ssp silvestris / ampelometry / microsatellite markers / resistance

\section{INTRODUCTION}

L'aire naturelle de répartition mondiale de l'espèce Vitis vinifera L. s'étend du Turkestan jusqu'à la façade atlantique européenne ainsi qu'en Afrique du Nord, autour de la Méditerranée. En France, à l'état spontané, c'est la seule espèce endémique représentante de la famille des Vitacées.

Pour les représentants sauvages de Vitis vinifera L. dénommés aussi « lambrusques », l'introduction au cours du XIX $^{\mathrm{e}}$ siècle de maladies et ravageurs en provenance d'Amérique du Nord (phylloxera surtout mais aussi oïdium, mildiou...), les accidents climatiques comme les gelées d'hiver, et les actions anthropiques (incendies, déforestation, disparition des haies ou du bocage, mise en place de barrages, remembrement, utilisation de désherbants, extensification de l'élevage, urbanisation) ont joué et continuent de jouer un rôle déterminant dans le processus d'appauvrissement.

L'introduction d'autres espèces du genre Vitis en relation avec l'utilisation des porte-greffe a pu également engendrer des risques non négligeables d'introgression génétique à travers les semis, notamment à proximité des vignobles. À ce niveau, étant donné les possibilités de pollinisations croisées, la détermination du statut exact des génotypes observés reste problématique car la différenciation entre lambrusques vraies (ou autochtones), lambrusques post-culturales (cépages abandonnés), lambrusques subspontanées (pépins de cépages), lambrusques coloniales (semis de lambrusques subspontanées) et lambrusques métisses (hybrides de lambrusques autochtones avec une des autres formes) est très difficile [12] sans compter les risques de confusion avec des hybrides d'espèces américaines.

Le compartiment sauvage, Vitis vinifera L. subsp. silvestris (Gmelin) Hegi, est donc dans ce contexte en voie de disparition et son aire de répartition en 
très forte régression. Les peuplements signalés [1] ne sont d'ailleurs souvent constitués que de quelques individus, très rarement de quelques dizaines.

Dans la perspective de la mise en place d'une action de conservation de ces ressources, l'approfondissement des connaissances concernant la constitution et la dynamique de ces populations devrait permettre de mieux raisonner l'échantillonnage au sein de peuplements et d'apprécier la possibilité de la conservation in situ. Une première étape du travail vise à mieux cerner la diversité rencontrée au sein d'un peuplement particulier que nous avons pris comme modèle, au travers de différentes approches (ampélographique, moléculaire, résistance au bioagresseurs). Afin de mieux cerner les relations entre cette population et le compartiment cultivé, nous avons également comparé la diversité moléculaire des lambrusques à celle d'un échantillon correspondant aux cépages autochtones ou cultivés actuellement dans la région.

\section{MATÉRIEL ET MÉTHODES}

\subsection{Matériel végétal}

Suite aux renseignements fournis par le Professeur Boubals (comm. pers.) et aux travaux préliminaires réalisés par C. Cuisset [9], nous avons choisi d'étudier de façon plus approfondie la population de lambrusques située sur les versants du Pic Saint-Loup, à proximité du village de Cazevieille dans l'Hérault (France). L'ensemble des individus recensés au cours des différentes prospections, soit quinze pieds de vignes sauvages, a été analysé. Ces accessions ont été numérotées : PSL1 à PSL15. Les proximités géographiques de ces individus permettent de distinguer 4 groupes (Tab. I) : les groupes 1 et 2 situés sur le versant sud et les groupes 3 et 4 situés sur le versant nord. Dans certains cas, du fait de leur proximité et des possibilités de marcottage, il était délicat, in situ, d'individualiser ou non certaines accessions ; c'est pourquoi, par précaution, quelques échantillons ont été subdivisés en « $a »$ et $《 b »$. Différents types de prélèvements ont été réalisés en 1998 : des jeunes feuilles pour l'extraction de l'ADN, des feuilles adultes pour l'analyse ampélométrique, et des boutures aoûtées pour l'évaluation de la tolérance aux bioagresseurs. Des observations et des notations complémentaires (villosité, sexe) ont également été réalisées sur place en 1997 et 1998 selon le code de descripteurs de la vigne proposé par l'IPGRI [11].

De plus, afin d'étudier au niveau moléculaire les relations éventuelles entre ces individus sauvages et le compartiment cultivé, des jeunes feuilles de 26 cépages présents dans la région ou autochtones (Tab. II) ont été prélevées dans la collection du Domaine de Vassal (INRA, 34340 Marseillan). 
Tableau I. Localisation géographique et résultats des notations réalisées pour deux descripteurs IPGRI sur la population de lambrusques du Pic Saint-Loup.

\begin{tabular}{lccc}
\hline Accessions & $\begin{array}{c}\text { Regroupement sur la } \\
\text { base de la proximité } \\
\text { microgéographique }\end{array}$ & Sexe des fleurs & $\begin{array}{c}\text { Densité des poils couchés entre } \\
\text { les nervures sur la face } \\
\text { inférieure de la feuille adulte }\end{array}$ \\
\hline PSL3 & 1 & Mâle & Nulle ou très faible \\
\hline PSL1 & 1 & Mâle & Faible \\
\hline PSL2 & 1 & Mâle & Faible \\
\hline PSL10 & 4 & Mâle & Faible \\
\hline PSL14 & 3 & Mâle & Faible \\
\hline PSL4a & 2 & Mâle & Moyenne \\
\hline PSL11 & 4 & Mâle & Moyenne \\
\hline PSL7 & 3 & Femelle & Nulle ou très faible \\
\hline PSL6 & 1 & Femelle & Faible \\
\hline PSL12 & 4 & Femelle & Faible \\
\hline PSL13 & 4 & Femelle & Faible \\
\hline PSL15 & 3 & Femelle & Faible \\
\hline PSL8 & 3 & Non déterminé & Nulle ou très faible \\
\hline PSL5 & 1 & Non déterminé & Faible \\
\hline & & & \\
\hline & & & \\
\hline
\end{tabular}

\subsection{Ampélométrie}

Dix feuilles adultes ont été échantillonnées et prélevées pour chaque accession. Elles ont été séchées et mises en herbier avant d'être analysées à l'aide d'une table à digitaliser (Graphtec KD 3300) reliée à un micro-ordinateur et du logiciel d'acquisition de données mis au point par Romani et al. [17]. Les coordonnées de 51 points par feuilles sont enregistrées. Elles permettent de générer 121 paramètres quantitatifs de longueurs, d'angles et de rapports ou de combinaisons qui définissent l'architecture et les principales caractéristiques des feuilles de vigne [8].

\subsection{Extraction de l'ADN et analyse microsatellite}

Les jeunes feuilles prélevées ont été rapidement congelées dans l'azote liquide et conservées à $-80^{\circ} \mathrm{C}$. L'extraction de l'ADN a été réalisée selon la méthode décrite par This et al. [19], à partir d'un gramme de matériel végétal. Dix locus microsatellites (VVMD5, VVMD6, VVMD7, VVMD17, VVMD24, VVMD25, 
Tableau II. Liste des cépages autochtones ou cultivés dans la région prélevés dans la collection du Domaine de Vassal (Inra, 34340 Marseillan).

\begin{tabular}{ll}
\hline Araignan B & Alicante Bouschet N \\
\hline Bourboulenc B & Aramon N \\
\hline Clairette B & Aspiran N \\
\hline Colombaud B & Aubun N \\
\hline Listan B & Bouteillan N \\
\hline Macabeu B & Brun argenté $\mathrm{N}$ \\
\hline Muscat à petits grains B & Calitor N \\
\hline Muscat d'Alexandrie B & Carignan N \\
\hline Pascal B & Cinsaut N \\
\hline Piquepoul B & Grenache N \\
\hline Terret G & Mourvèdre N \\
\hline Ugni blanc B & Eillade N \\
\hline & Petit Bouschet $\mathrm{N}$ \\
\hline
\end{tabular}

VVMD27, VVMD28, VVMD32, VVMD36) ont été testés [5,6], et leur analyse a été réalisée sur gel d'acrylamide dénaturant avec révélation au nitrate d'argent selon le protocole décrit par Loureiro et al. [13]. Les différents allèles ont été déterminés par comparaison avec des marqueurs de taille et avec des allèles de cépages connus, servant de référence. Les données ont été enregistrées sous forme d'une matrice de type présence - absence de chaque allèle aux dix locus. Les individus présentant une seule bande pour un locus donné, ont toujours été considérés comme homozygote dans la mesure où il n'était pas possible de vérifier la présence d'un allèle nul. Dans ce cas, la présence de cet allèle a été alors prise en compte deux fois.

\subsection{Tests de résistance}

Les bois prélevés ont été conservés en chambre froide $\left(4^{\circ} \mathrm{C}\right)$ pendant deux mois, puis des boutures à deux yeux ont été réalisées et mises en serre. Les sarments étant très faibles, seules 11 accessions se sont développées avec seulement une à sept boutures disponibles par accession.

\subsection{1. Ö̈dium}

Des feuilles encore en croissance mais étalées ont été prélevées sur les boutures pour obtenir trois rondelles $(1,7 \mathrm{~cm}$ de diamètre) à l'aide d'un 
emporte-pièce. Elles sont déposées en boîte de Petri sur milieu gélosé $\left(10 \mathrm{~g}^{-1}\right.$ d'agar-agar, $30 \mathrm{mgl}^{-1}$ de benzimidazole) avec trois rondelles de feuilles de Cabernet-Sauvignon comme témoin. L'inoculum est obtenu à partir de jeunes feuilles de Cabernet-Sauvignon préalablement désinfectées ( 5 min dans $\mathrm{CaCl} 2$ à $40 \mathrm{~g} \mathrm{l}^{-1}$ ) et lavées dans de l'eau stérile puis infectées à l'aide d'une souche monoconidienne d'oïdium clonée par repiquages successifs. Deux à trois feuilles infectées sont utilisées pour inoculer 7 boîtes de Petri disposées à la base d'une tour d'inoculation [7]. Quatre lames gélosées sont réparties entre les boîtes pour pouvoir vérifier par comptage que la densité de conidies déposées se situe entre 200 et 500 par $\mathrm{cm}^{2}$. Les boîtes sont ensuite placées en chambre de culture $\left(25^{\circ} \mathrm{C}+/-1{ }^{\circ} \mathrm{C}, 50 \mu \mathrm{e} / \mathrm{sm} 2,15 \mathrm{~h} / \mathrm{j}\right.$ d'éclairement, $70 \%$ d'hygrométrie $)$. Le développement du champignon est observé 10 jours après et les plantes sont notées sensibles lorsqu'il y a présence de conidies.

En complément, les symptômes de la maladie ont été aussi observés sur les boutures en serre, en fin de végétation, après arrêt des traitements et développement d'oïdium (souche non contrôlée).

\subsubsection{Phylloxera}

Le test a été réalisé suivant le protocole décrit par Boubals [2]. Des feuilles couvertes de galles ont été prélevées sur des feuilles de porte-greffe et enfouies dans les pots des boutures à tester. Cette opération a été réalisée début juillet et renouvelée mi-août. Les plants ont été dépotés à la fin de l'automne pour observer la présence ou non de tubérosités et de nodosités.

\subsection{Analyse des données}

\subsubsection{Données ampélométriques}

Soixante et un paramètres de longueur, d'angle et de rapports de longueur et d'angle ont été calculés pour les 15 individus pour les 10 feuilles analysées. Une analyse de variance effectuée à l'aide de la procédure GLM du logiciel de statistiques édité par SAS ${ }^{\circledR}$ Institute, a permis d'estimer l'effet génotype sur ces paramètres et ainsi d'évaluer la fiabilité de leur utilisation. Trente cinq de ces variables quantitatives ont été retenues pour une analyse en composantes principales, sur la base de résultats d'études antérieures dans ce domaine $[8,14]$. L'analyse en composantes principales a été effectuée à l'aide du logiciel SAS ${ }^{\circledR}$ sur les valeurs moyennes de chaque caractère.

\subsubsection{Données moléculaires}

Les données recueillies sur l'ensemble des 41 individus de l'étude ont été traitées par analyse en composantes principales non normée à l'aide du logiciel Statlab. Pour ce faire, les données moléculaires ont été codées en présence/absence 
pour chacun des allèles présents dans l'échantillon (soit 80 allèles). Puis, nous avons doublé les colonnes pour lesquelles au moins un individu était homozygote. La matrice des données comprenait donc 41 lignes et 115 colonnes. Cette opération transforme les données en données quantitatives discontinues et l'utilisation d'une ACP non normée se justifie dans la mesure où les sommes des données par ligne sont identiques (M. Dauzard, communication personnelle).

D'autre part, des analyses complémentaires ont été conduites sur les données recueillies pour les individus sauvages du Pic Saint-Loup. Dans un premier temps, un test d'écart à la panmixie a été effectué à l'aide du logiciel Genepop3 [16]. Des distances génétiques ont ensuité été calculées : la distance d'allèles partagés ("Shared allele distance" [4]) a été calculée à l'aide du logiciel développé par J. Brzutowski (adresse web : http://gause.biology.ualberta. ca/ jbrzusto/sharedst.html) et des indices de similarité de Nei [15] ont été calculés à l'aide du logiciel SSR (W. Lamboy, communication personnelle). À partir de la distance d'allèles partagés, un arbre a été défini à l'aide de l'algorithme NJ-tree [18] grâce au logiciel Phylip [10].

\section{RÉSULTATS}

\section{1. Étude morphologique}

Le tableau I indique les résultats des descriptions ampélographiques portant sur le sexe des fleurs et la densité des poils couchés entre les nervures de la face inférieure de la feuille adulte. Le sexe des individus sauvages décrits dans cette étude est séparé. Ainsi, on note un pourcentage important de pieds mâles (58\% des plants pour lesquels ce caractère a pu être observé). Ils se répartissent avec les pieds femelles dans les 4 groupes géographiques. La villosité ne semble pas discriminer les individus, que ce soit par rapport à la position géographique ou en relation avec le sexe même si les 2 seuls individus notés à villosité moyenne sont mâles ; l'échantillon étant trop faible pour en tirer des conclusions significatives.

En ce qui concerne l'analyse des données ampélométriques, tous les paramètres sauf trois ( $\mathrm{SP}$ profondeur du sinus pétiolaire ; $\mathrm{F}$ : angle de la nervure principale et la dent de son extrémité ; D : angle des nervures pétiolaires principales et secondaires) ont montré un effet génotype hautement significatif. La variabilité est donc bien liée à l'effet du génotype. L'analyse en composantes principales n'a porté que sur trente cinq des variables quantitatives sélectionnées sur la bases d'études préliminaires $[8,14]$. Les résultats font apparaître que les 4 premiers axes permettent d'expliquer $76 \%$ de la diversité globale. 


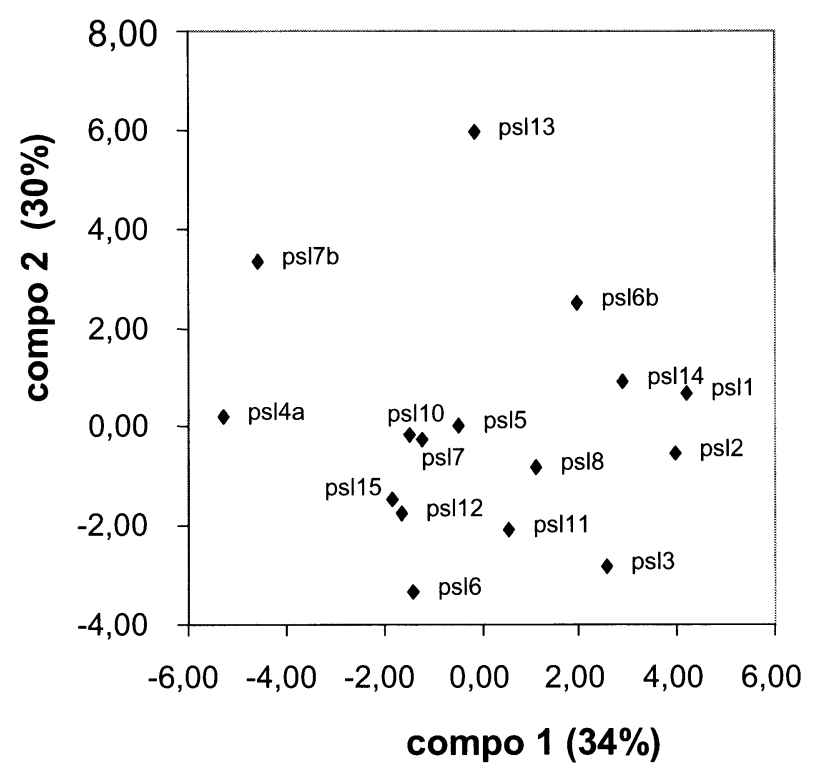

Figure 1. Représentation de la dispersion des lambrusques du Pic Saint-Loup selon les axes 1 et 2 de l'analyse en composantes principales réalisée à partir de 35 variables ampélométriques.

Les variables qui participent le plus aux deux premiers axes sont respectivement :

- les profondeurs des sinus latéraux supérieurs et inférieurs (paramètres U et $\mathrm{O}$ ) qui s'opposent aux variables constituées par les rapports entre les angles des nervures principales et les sinus latéraux $(\mathrm{A} / \mathrm{U}, \mathrm{B} / \mathrm{O}$, $\mathrm{A}+\mathrm{B} / \mathrm{U}+\mathrm{O})$

- la largeur de la feuille (AN2) et la longueur de la dent terminant la nervure N4 (L4) qui s'opposent aux variables constituées par les rapports entre les sinus latéraux et les nervures principales adjacentes $(\mathrm{O} / \mathrm{N} 3$, $\mathrm{U}+\mathrm{O} / \mathrm{N} 2+\mathrm{N} 3)$.

Au niveau des individus (Fig. 1) trois d'entre eux (PSL4a, PSL7b et PSL13) semblent se démarquer quelque peu du cœur de la population. Par ailleurs, les pieds mâles se répartissent préférentiellement selon l'axe 1 alors que les individus femelles se dispersent selon l'axe 2 . On peut également remarquer les positions relativement individualisées des échantillons PSL5, PSL6 et PSL6b d'une part et des échantillons PSL7 et PSL7b d'autre part. 
Tableau III. Résultats synthétiques de l'analyse des 41 individus (lambrusques et cépages) étudiés sur dix locus microsatellites.

\begin{tabular}{|c|c|c|c|c|c|c|c|c|}
\hline \multirow[b]{2}{*}{ Locus } & \multirow[b]{2}{*}{ 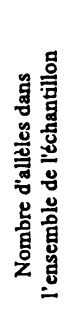 } & \multirow[b]{2}{*}{ 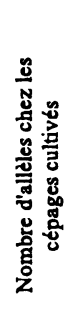 } & \multirow[b]{2}{*}{  } & \multirow[b]{2}{*}{ 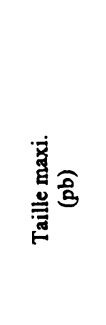 } & \multirow[b]{2}{*}{ 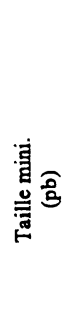 } & \multicolumn{3}{|c|}{$\%$ de plantes homozygotes } \\
\hline & & & & & &  &  & 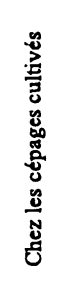 \\
\hline VVMDS & 7 & $7(3)$ & 4 & 240 & 226 & 31,7 & 26,7 & 34,6 \\
\hline VVMD6 & 5 & $5(1)$ & 4 & 211 & 191 & 19,5 & 26,7 & 15,4 \\
\hline VVMD7 & 9 & $7(5)$ & $4(2)^{*}$ & 264 & 234 & 34,5 & 53,3 & 23,1 \\
\hline VVMD17 & 6 & $6(2)$ & 4 & 236 & 212 & 31,7 & 40 & 26,9 \\
\hline VVMD24 & 7 & $7(3)$ & 4 & 218 & 207 & 29,3 & 33,3 & 26,9 \\
\hline VVMD25 & 8 & $7(2)$ & $6(1)$ & 272 & 242 & 12,2 & 6,7 & 15,4 \\
\hline VVMD27 & 7 & $7(4)$ & 3 & 194 & 179 & 22 & 46,7 & 7,7 \\
\hline VVMD28 & 10 & $9(6)$ & $4(1)$ & 274 & 230 & 26,8 & 46,7 & 15,4 \\
\hline VVMD32 & 8 & $8(3)$ & 5 & 272 & 240 & 22 & 33,3 & 15,4 \\
\hline VVMD36 & 13 & $11(6)$ & $7(2)$ & $297(400)$ & 243 & 17,1 & 26,7 & 11,5 \\
\hline
\end{tabular}

\subsection{Analyse microsatellite}

\subsubsection{Diversité allélique}

L'analyse des 41 individus (lambrusques et cépages cultivés) à l'aide des 10 locus microsatellites a révélé un nombre d'allèles variant de 5 à 13 par locus (Tab. III). Le nombre d'allèles observés dans la population sauvage est beaucoup plus faible puisque 3 à 7 allèles seulement par locus ont été observés. Pour quatre locus (VVMD7, VVMD25, VVMD28 et VVMD36), 1 à 2 allèles étaient spécifiques de la population sauvage. Le pourcentage de plantes homozygotes varie de 12,2 à $34,5 \%$ dans l'échantillon total mais il est beaucoup plus élevé dans la population sauvage (26.7 à $53.3 \%$ ) notamment pour 7 locus. Le pourcentage d'hétérozygotie par plante chez les lambrusques varie de 50 à $100 \%$ avec une moyenne de $66.2 \%$ (Tab. IV), tandis que l'hétérozygotie moyenne au sein des cépages cultivés est de $80.8 \%$. Le test de Hardy-Weinberg révèle par ailleurs que la population est à l'équilibre $(\mathrm{P}$-val $=0.3804$ pour le test de l'excès d'hétérozygotes). 
Tableau IV. Taux d'hétérozygotie chez les individus de la population du pic Saint-Loup.

\begin{tabular}{lc}
\hline Accessions & $\begin{array}{c}\% \\
\text { hétérozygote }\end{array}$ \\
\hline PSL1 & 50 \\
\hline PSL2 & 70 \\
\hline PSL3 & 80 \\
\hline PSL4a & 100 \\
\hline PSL5 & 50 \\
\hline PSL6 & 50 \\
\hline PSL7 & 80 \\
\hline PSL10 & 70 \\
\hline PSL11 & 50 \\
\hline PSL12 & 90 \\
\hline PSL13 & 60 \\
\hline PSL14 & 60 \\
\hline PSL15 & 50 \\
\hline \hline Moyenne & 66.15 \\
\hline
\end{tabular}

\subsubsection{Relation entre les compartiments}

Les résultats de l'analyse en composantes principales font apparaître que les 3 premiers axes permettent d'expliquer $32 \%$ de la diversité globale. Le plan des axes 1 et 2 (Fig. 2) montre une très nette séparation des individus sauvages par rapport aux individus cultivés qu'il s'agisse des cépages actuellement cultivés dans la zone (Carignan, Grenache...) ou des vieux cépages autochtones (Aramon, Aspiran...). Aucun individu du Pic Saint-Loup ne peut ainsi résulter d'un croisement avec un des 21 cépages autochtones ou actuellement cultivés, ce que confirme la recherche des individus partageant des allèles communs aux différents locus. On peut noter également que le nuage des cépages cultivés est relativement plus compact que celui composé des individus sauvages.

\subsubsection{Structuration de la diversité chez les lambrusques}

$\mathrm{Au}$ sein de la population sauvage du Pic Saint-Loup, seuls les individus PSL3a et PSL3b d'une part et PSL5, PSL6 et PSL6b d'autre part ne sont pas différenciés. Les autres individus présentent tous des profils distincts aux 10 locus. En ne considérant que les individus sauvages distincts, les indices 
Tableau V. Résultats synthétiques des tests de tolérance aux bioagresseurs.

\begin{tabular}{|c|c|c|c|c|}
\hline Accessions & $\begin{array}{l}\text { Test oïdium } \\
\text { in vitro }\end{array}$ & $\begin{array}{l}\text { Test oïdium } \\
\text { en serre }\end{array}$ & $\begin{array}{l}\text { Test phylloxera } \\
\text { tubérosités }\end{array}$ & $\begin{array}{l}\text { Nombre de } \\
\text { plantes testées }\end{array}$ \\
\hline PSL1 & S & S & 1 & 1 \\
\hline PSL2 & $+/-S$ & $+/-\mathrm{S}$ & 1 & 2 \\
\hline PSL4a & $\mathrm{S}$ & $S$ & 0 & 2 \\
\hline PSL4b & $\mathrm{S}$ & $\mathrm{S}$ & 1 & 1 \\
\hline PSL5 & $S$ & $S$ & 1 & 2 \\
\hline PSL6 & $S$ & $\mathrm{~S}$ & 1 & 1 \\
\hline PSL7 & $\mathrm{S}$ & $S$ & 1 & 4 \\
\hline PSL8 & $S$ & $\mathrm{~S}$ & 1 & 4 \\
\hline PSL10 & $\mathrm{S}$ & $\mathrm{S}$ & 1 & 7 \\
\hline PSL12 & $S$ & $S$ & 1 & 2 \\
\hline PSL13 & $\mathrm{S}$ & $\mathrm{S}$ & 1 & 1 \\
\hline
\end{tabular}

de similarité de Nei [15] sont compris entre 0,2 et 0,85 . Les individus les plus distants étant PSL15 et PSL5 (PSL6), et les plus proches, PSL10 et PSL13. Par ailleurs, les individus PSL2 et PSL4, PSL3 et PSL4, PSL10 et PSL13, PSL14 et PSL3, PSL15 et PSL7 partagent au moins un allèle commun sur chacun des 10 locus analysés. Ces relations sont confirmées par l'arbre dressé à partir de la distance d'allèles partagés (Fig. 3). En effet, les individus sont regroupés en trois clades bien distinctes qui englobent ces relations généalogiques possibles et qui peuvent également se rapprocher des groupes microgéographiques.

\subsection{Tests de résistance à l'oïdium et au phylloxera}

Les résultats des tests sont reportés dans le tableau V. Il faut signaler que la faible vigueur des plantes a été un facteur limitant pour l'ensemble des tests artificiels. L'ensemble des génotypes paraît sensible à l'oïdium. Cependant, aussi bien in vitro qu'en serre, le champignon s'est nettement moins bien développé sur PSL2 que sur les autres accessions et que sur le Cabernet-Sauvignon (témoin), et ce malgré une vigueur correcte des plantes PSL2.

L'ensemble des génotypes semble également être sensible au phylloxera. Seul PSL4a présentait peu de symptômes (notamment peu de tubérosités) malgré, là aussi, un système racinaire bien développé. 


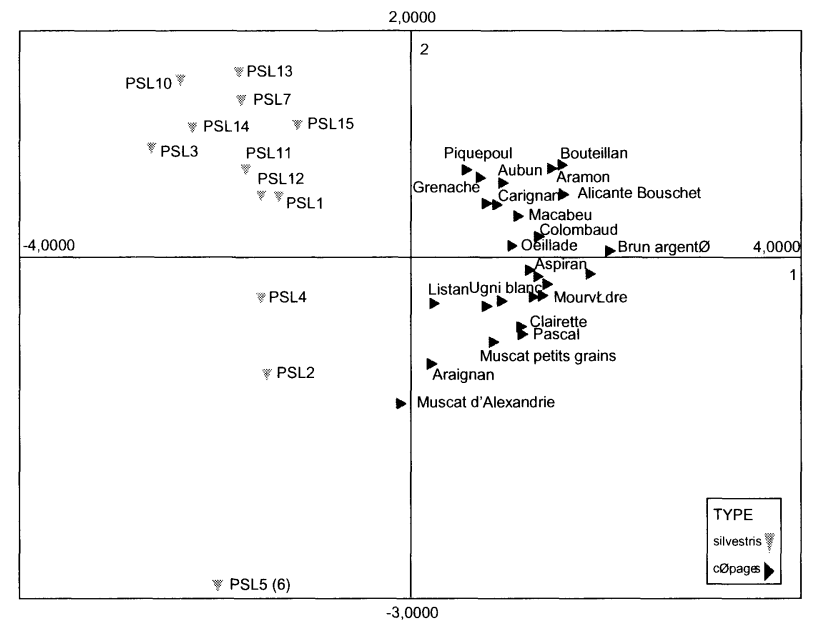

Figure 2. Représentation de la dispersion des individus sauvages (silvestris) et cultivés (cépages) selon les axes 1 et 2 de l'analyse en composantes principales non normée, réalisée sur les données moléculaires obtenues à partir de l'étude de 10 locus microsatellites.

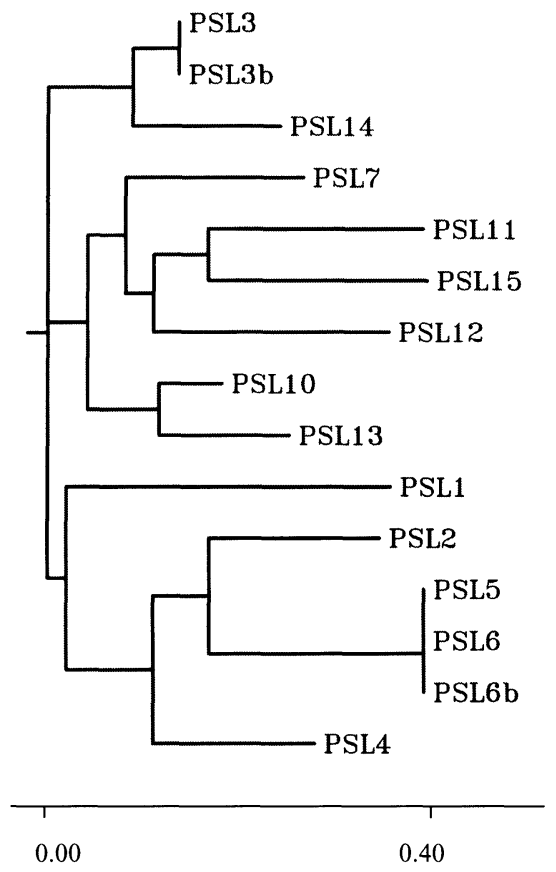

Figure 3. Arbre obtenu à l'aide de l'algorithme NJ-tree, à partir des distances d'allèles partagés calculées pour les 10 locus microsatellites. 


\section{DISCUSSION}

\subsection{Variabilité morphologique et intérêt des descripteurs}

Les descriptions et les analyses effectuées sur cette population de lambrusques permettent de faire ressortir et de sélectionner les caractères les plus importants pour décrire la diversité rencontrée.

Le sexe peut bien évidemment être considéré comme un caractère important puisque la quasi-totalité des cépages cultivés sont hermaphodites ou plus rarement femelles [3] alors que la plupart des lambrusques de notre étude sont diö̈ques. La villosité est également un caractère morphologique à prendre en compte pour évaluer la diversité (les lambrusques sont glabres), d'autant que cette notation, comme celle du sexe, peut se réaliser directement sur place.

En ce qui concerne les descripteurs ampélométriques, nous retiendrons préférentiellement, sur la base des résultats de l'analyse en composantes principales, les descripteurs complémentaires suivants : la profondeur des sinus latéraux $(\mathrm{O}, \mathrm{U})$, le degré d'ouverture des nervures principales $(\mathrm{A}, \mathrm{B})$, la largeur de la feuille (AN2, N2, N3) et la longueur des dents (L4). On retrouve ainsi en partie les descripteurs généralement utilisés pour décrire habituellement les individus du compartiment cultivé [8] et, par rapport à l'ensemble des paramètres mesurés, le nombre plus limité de caractères proposés permet en définitive d'envisager leur utilisation pour décrire un plus grand nombre d'individus.

Les distances entre les barycentres des individus PSL5, PSL6 et PSL6b (Fig. 1) alors que l'analyse moléculaire a montré qu'ils étaient identiques traduit l'effet de l'environnement sur les caractères ampélométriques, ce qui explique leur utilisation relativement limitée en terme d'identification. Par contre, leur prise en compte en vue de décrire la diversité peut être envisagée comme une approche complémentaire très intéressante. Ainsi dans la perspective d'un échantillonnage au sein de cette population, on pourrait envisager une sélection des individus en combinant la répartition géographique, les descripteurs liés au sexe et à la villosité et les résultats des analyses ampélométriques. Les lambrusques PSL13, PSL6, PSL4a, PSL1 et PSL7b constitueraient alors de bons candidats.

\subsection{Diversité moléculaire et relations avec le compartiment cultivé}

La nette séparation entre les deux compartiments sauvage et cultivé confirme bien les premiers résultats que nous avions obtenus à l'aide de marqueurs RAPD [9]. Cette différenciation repose essentiellement sur des allèles spécifiques puisque 4 allèles sur les 80 sont spécifiques des sauvages et 35 allèles sont spécifiques des cépages cultivés. Le compartiment cultivé présente une richesse allélique plus grande. Par contre, dans l'analyse en composantes principales, le nuage des individus cultivés est plus regroupé. Ces données confirment donc 
l'intérêt de ce matériel sauvage dans le cadre des ressources génétiques de la vigne puisqu'il représente un compartiment distinct. Des études élargies avec des peuplements d'origines géographiques diversifiées permettront de préciser cet aspect.

$\mathrm{Au}$ sein de la population de lambrusques du Pic Saint-Loup, les individus sont tous différents sauf PSL3 et PSL3b, que nous supposions identiques, et PSL5, PSL6 et PSL6b qui s'avèrent être les mêmes individus résultant vraisemblablement d'un phénomène de marcottage naturel. Cette identité a été détectée par l'analyse moléculaire, ce qui souligne l'intérêt de cette approche. De plus, cette accession PSL5 (PSL6) semble être assez différente des autres lambrusques.

Dans la mesure où l'on voudrait échantillonner les individus sauvages les plus diversifiés à l'aide des résultats moléculaires (distances, A.C.P., arbre) obtenus à partir des données moléculaires, un individu par clade sur l'arbre pourrait alors être choisi.

Le test de Hardy-Weinberg montre que nous avons une population à l'équilibre. Un pourcentage plus faible de marqueurs sont à l'état homozygote par rapport aux cépages cultivés. Bien que la vigne se reproduise naturellement de façon végétative par marcottage (ce que nous avons également pu observer), la reproduction sexuée existe et les données microsatellites font apparaître des relations généalogiques entre les différents individus. Bien que des analyses complémentaires soient nécessaires pour vérifier ces parentés possibles, il semble cependant que cette population évolue par voie sexuée et des croisements pourraient être possibles même entre les individus situés sur des versants différents. Il ne semble cependant pas y avoir eu de transfert récent avec le compartiment cultivé, puisque aucun des cépages plantés à proximité ne semble être un parent direct. Des analyses plus approfondies de descendances issues des individus de cette population seront très intéressantes pour confirmer ce point.

\subsection{Tolérance aux bioagresseurs}

Au cours de cette première approche l'ensemble des accessions récoltées sur le Pic Saint-Loup semble donc globalement sensible à l'oïdium et au phylloxera, ce qui n'est pas surprenant dans la mesure où tous les représentants connus de Vitis vinifera le sont. Ainsi ces plantes ont sans doute davantage résisté grâce à leur isolement relatif et à une faible pression d'inoculum dans cet environnement particulier. Cependant, les tests réalisés étaient de nature qualitative et il serait nécessaire d'analyser à nouveau plus précisément ces accessions afin de mettre en évidence d'éventuels niveaux de tolérance à l'oïdium (notamment pour PSL2) ou au phylloxera (pour PSL4a), qui pourraient s'avérer intéressants. Cela justifierait alors pleinement de prendre en considération ces accessions dans la perspective d'un échantillonnage. Cependant, pour réaliser ces tests, une des difficultés réside dans l'obtention de plantes présentant un 
état physiologique correct et homogène aussi bien au niveau de la partie aérienne que racinaire afin de pouvoir analyser de façon quantitative leur niveau de sensibilité et pour permettre des comparaisons plus précises.

En conclusion, nous avons caractérisé les individus sauvages de la population du Pic Saint-Loup à l'aide de données géographiques, morphologiques et de données moléculaires. Ces approches sont tout à fait complémentaires et permettent de mieux cerner la diversité du pool sauvage. Elles permettent également d'envisager un échantillonnage des individus les plus intéressants. Le caractère du sexe est spécifique pour cette population mais il serait intéressant de caractériser des individus sauvages hermaphrodites pour confirmer l'importance de ce caractère comme test initial. De même, une analyse plus approfondie d'autres peuplements sauvages devra également être entreprise pour confirmer ces données et permettra notamment de comparer divers peuplements français.

\section{REMERCIEMENTS}

Nous remercions le Bureau des ressources génétiques (BRG) pour le soutien financier accordé à ces recherches. Nous tenons également à remercier vivement le Professeur Boubals et Cécile Cuisset pour leur contribution déterminante dans l'initiation de ces travaux.

\section{RÉFÉRENCES}

[1] Arnold C., Gillet F., Gorat J.M., Situation de la vigne sauvage Vitis vinifera ssp. silvestris en Europe, Vitis 37 (1998) 159-170.

[2] Boubals D., Étude de la distribution et des causes de la résistance au phylloxera radicicole chez les Vitacées, Ann. Amél. Plantes 16 (1966) 145-184.

[3] Boursiquot J.M., Dessup M., Rennes C., Distribution des principaux caractères phénologiques, agronomiques et technologiques chez Vitis vinifera L., Vitis 34 (1995) 31-35.

[4] Bowcock A.M., Ruiz-Linares A., Tomfohrde J., Minch E., Kidd J.R., CavalliSforza L.L., High resolution of human evolution with polymorphic microsatellites, Nature 368 (1994) 455-457

[5] Bowers J.E., Dangl G.S., Vignani R., Meredith C.P., Isolation and characterization of new polymorphic simple sequence repeat loci in grape (Vitis vinifera L.) Genome 39 (1996) 628-633.

[6] Bowers J.E., Dangl G.S., Meredith C.P., Development and characterization of additional microsatellite DNA markers for grape, Am. J. Enol. Vitic. 50 (1999) 243-246.

[7] Cartolaro P., Stéva H., Maîtrise de l'oïdium au laboratoire, Phytoma, 419 (1990) 37-40.

[8] Cid-Alvarez N., Boursiquot J.M., Saa-Otero M.P., Romani-Martinez L., Différenciation des cépages autochtones du Nord-Ouest de l'Espagne (Galice) et 
élaboration d'une clé de détermination basée sur l'ampélométrie, J. Int. Sci. Vigne Vin 28 (1994) 1-17.

[9] Cuisset C., Étude de la diversité génétique de la vigne (Vitis vinifera L.) par des marqueurs morphologiques et moléculaires, Thèse, ENSA Montpellier, 1998.

[10] Felsenstein, J. PHYLIP - Phylogeny Inference Package (Version 3.2). Cladistics 5 (1989) 164-166.

[11] I.P.G.R.I., Descripteurs de la vigne (Vitis spp.), Rome, 1997.

[12] Levadoux L., Les populations sauvages et cultivées de Vitis vinifera L., Ann. Amél. Plantes, 6 (1956) 59-118.

[13] Loureiro D., Martinez C., Boursiquot J.M., This P., Molecular marker analysis of Vitis vinifera "Albariño" and some similar grapevine cultivars. J. Amer. Soc. Hort. Sci., 123 (1998) 842-848.

[14] Martinez M.C., Boursiquot J.M., Grenan S., Boidron R., Étude ampélométrique de feuilles adultes de somaclones du cv. Grenache N (Vitis vinifera L.), Can. J. Bot. 75 (1997) 333-345.

[15] Nei M., Genetic distance between populations, American Naturalist, 106 (1972) 283-292.

[16] Raymond M., Rousset F. Genepop: population genetics software for exact tests and eumenicism. J. Hered., 86 (1995) 248-249.

[17] Romani L., Legido J.L., Saa M.P., Cid N., Desing and updating of a computer system for the ampelometric characterisation of grapevine varieties, J. Wine Research, 3 (1993) 197-203.

[18] Saitou N., Nei M., The neighbor-joining method: a new method for reconstructing phylogenetic trees, Mol. Biol. Evol. 4 (1987) 406-425.

[19] This P., Cuisset C., Boursiquot J.M., Development of stable molecular markers based on RAPD for the identification of grapevine rootstocks, Am. J. Enol. Vitic. 48 (1997) 492-501. 